\title{
LLUVIAS TORRENCIALES Y CAMBIOS GEOMORFICOS EN UNA PEQUEÑA CUENCA DE MONTAÑA: EL BARRANCO DE LA CUESTA DE LA VEGA (VALENCIA)
}

\author{
Alejandro J. Pérez Cueva* \\ Adolfo Calvo Cases*
}

\begin{abstract}
RESUMEN
Se estudian los efectos de unas lluvias torrenciales de gran magnitud $-450 \mathrm{~mm}$. - así como fuertes y prolongadas intensidades $\longrightarrow 90 \mathrm{~mm}$. durante $3 \mathrm{~h}$.- en una pequeña cuenca mediterránea (Ayora, Valencia). Allí, las lluvias produjeron unos flujos extraordinarios -entre 11,2 y 38,2 $\mathrm{m}^{3} / \mathrm{seg} / \mathrm{km}^{2}$ - y la reactivación de procesos de erosión y agradación, especialmente en valles de fondo plano con cultivos en terrazas, en los que llegaron a excavarse nuevos cauces. Se distinguen varios tipos de depósitos y de fenómenos erosivos y se discuten sus factores; entre ellos destacan la amplitud del valle, las pendientes locales, el abandono de los campos de cultivo (en sentido positivo) o el mismo aterrazamiento del valle.
\end{abstract}

\section{SUMMARY}

In this paper the effects of great magnitude $-450 \mathrm{~mm}$ - - as well as high continued intensity $\longrightarrow \geq 90 \mathrm{~mm}$. for $3 \mathrm{~h}$.- - storm-rains have been studied for a small mediterranean drainage basin (Ayora, Valencia). Such rains caused anormal flows - between 11.2 y $38.2 \mathrm{~m}^{3} / \mathrm{sec} / \mathrm{km}^{2}$ - and the reactivation of erosional and aggradational processes, especially at flat bottomed valleys with terrace culture, where new channels were formed. Several types of deposits and erosional phenomena are established; among them valley amplitude, local slopes, culture abandonment (in a positive sense), and valley floor terracing are pointed out.

* Departamento de Geografía. Universidad de Valencia. 


\section{ALEJANDRO PEREZ CUEVA, ADOLFO CALVO CASES}

En ambientes mediterráneos secos, donde sólo con periodicidad interanual y en un corto período de tiempo los cauces son vehículo de las aguas de escorrentía, la activación de los procesos fluviales se produce preferentemente en el marco de precipitaciones extraordinarias. Además, la activación se opera a menudo en un medio modificado por el hombre, que tiende a aprovechar como campos de secano todas las tierras y en especial los fondos de valle, al ser éstos dos lugares de mayor concentración de humedad. La región de Ayora, al igual que gran parte del Levante español, participa en buena medida de estas características.

En ella el temporal de octubre de 1982 llegó a producir importantes volúmenes de escorrentía y significativos cambios morfológicos. Este artículo pretende ejemplificar los cambios acaecidos en los cauces de una pequeña cuenca de drenaje como consecuencia de este temporal. Para ello, tras una descripción del marco natural sobre el que van a actuar los procesos, se aborda el problema de la evaluación de los caudales que en última instancia son los agentes responsables de la actividad en la cuenca. Por último, en base a observaciones y medidas de campo se describen las formas resultantes, dejando para el final una evaluación de los factores que han condicionado la respuesta morfológica.

La formación de un cauce nuevo en lugares donde no existía canal de drenaje definido debido a la actuación antrópica ha sido el proceso más relevante. Las características del lugar de emplazamiento, un valle de fondo plano colmatado por aportes fundamentales finos, y las formas de cauce resultantes, de fondo plano, paredes abruptas y fuerte gradiente longitudinal, recuerdan en gran medida a los arroyos del SW americano estudiados, entre otros, por COOKE y REEVES (1976) y LEOPOLD (1978). No obstante, más allá de las coincidencias morfológicas y dinámicas que ambos lugares tienen en común, existen en nuestro caso factores peculiares a la hora de explicar el proceso de incisión. Entre ellos la reducción de los fondos de valle a una sucesión de terrazas escalonadas va a ser de primordial importancia.

\section{El marco natural}

El barranco de la Cuesta de la Vega es un tributario del río Reconque situado al W de Ayora que forma parte de la red de drenaje del río Júcar. Con una superficie de $6,4 \mathrm{~km}^{2}$ su cuenca está labrada sobre materiales del Jurásico, Cretácico y Mio-Plioceno. Dominan en general las facies conglomeráticas de estos últimos períodos, junto con calizas cristalinas coniacienses-santonienses (área de Cerro Gordo) y calizas y calcoarenitas del Kimmeridgiense (área de la Casilla).

Se trata de una cuenca de relieve suave, con un desnivel máximo de 280 m. y una altitud media en torno a $740 \mathrm{~m}$. El 59,9\% de la superficie tiene pen- 


\section{LLUVIAS TORRENCIALES Y CAMBIOS GEOMORFOLOGICOS}

dientes por debajo de $7^{\circ}$, el $43 \%$ entre $7^{\circ}$ y $14^{\circ}$ y sólo el $5,8 \%$ por encima de $14^{\circ}$. En la distribución de estas pendientes (fig. 1B) destaca el que los valores moderados tiendan a situarse en torno a la divisoria de aguas, mientras que los tramos abruptos se hallan próximos al cauce principal y afluentes mayores. Ello es debido a que la red de drenaje actual se ha encajado sobre un antiguo nivel de erosión, probablemente villafranquiense, del que quedan abundantes retazos en el perímetro de la cuenca, especialmente en su porción meridional.

El barranco de la Cuesta de la Vega es, según la clasificación de Strahler, un cauce de $4 .{ }^{\circ}$ orden (1), al que afluyen cinco cuencas de $3 .{ }^{\text {er }}$ orden, dos de $2 .^{\circ}$ con una extensión equivalente a las de orden 3 , y un número elevado de pequeñas cuencas de $2 .^{\circ}$ y $1 .^{\text {er }}$ orden (fig. 1A). La red de afluentes adopta una forma de espiga respecto al cauce principal, si bien los de la margen izquierda son claramente más cortos, lo cual es reflejo de la disimetría de la cuenca.

Se han seleccionado 4 índices morfométricos como los más representativos (densidad de drenaje-Dd-, razón de bifurcación-Rb-, razón de relieveRr- y razón de elongación-Re-, vid. CHRISTOFOLETTI, 1971). La densidad de drenaje es moderadamente elevada y uniforme en el conjunto de cuencas. Los materiales calcáreos tienden a disminuir su valor, mientras que los materiales blandos terciarios y las zonas con fuerte impronta estructural dan valores más elevados. La razón de elongación es muy acentuada en todas las cuencas de la margen derecha lo que indica la débil jerarquización de la red formada sobre el glacis villafranquiense. La razón de relieve es moderadamente baja; su fuerte relación con el índice anterior indica factores genéticos similares. Por último, la razón de bifurcación marca amplias diferencias entre las distintas subcuencas, siendo muy elevada en las dos grandes cuencas de $2 . .^{\circ}$ orden y moderada en las cuencias elongadas de tercer orden, todas ellas labradas sobre el glacis villafranquiense; en el resto de cuencas de orden $3 .^{\circ}$ su valor es bajo (vid. tabla I).

TABLA I

Características morfométricas de las principales cuencas de drenaje

\begin{tabular}{crcrcrrrr} 
& A & B & C & D & E & F & G & \multicolumn{1}{c}{ H } \\
& & & & & & & & \\
$\mathrm{S}\left(\mathrm{Km}^{2}\right)$ & 6.41 & 1.2 & 0.44 & 0.66 & 0.7 & 0.36 & 0.36 & 0.54 \\
$\mathrm{Dd}$ & 6.25 & 6.45 & 6.73 & 6.00 & 5.54 & 7.31 & 8.17 & 4.67 \\
$\mathrm{Re}$ & 0.63 & 0.46 & 0.45 & 0.40 & 0.47 & 0.72 & 0.54 & 0.92 \\
$\mathrm{Rr}$ & 62.97 & 60.14 & 64.67 & 85.77 & 85.42 & 115.95 & 121.42 & 102.22 \\
$\mathrm{Rb}$ & 5.06 & 5.16 & 11.00 & 11.00 & 3.16 & 3.00 & 4.25 & 2.75
\end{tabular}

1. El trazado de la red ha sido realizado sobre una restitución fotogramétrica a escala 1/ 10.000 , a partir de fotogramas aéreos a escala aproximada $1 / 33.000$, considerando como cauce a concavidades o vaguadas con longitud superior a $50 \mathrm{~m}$. 


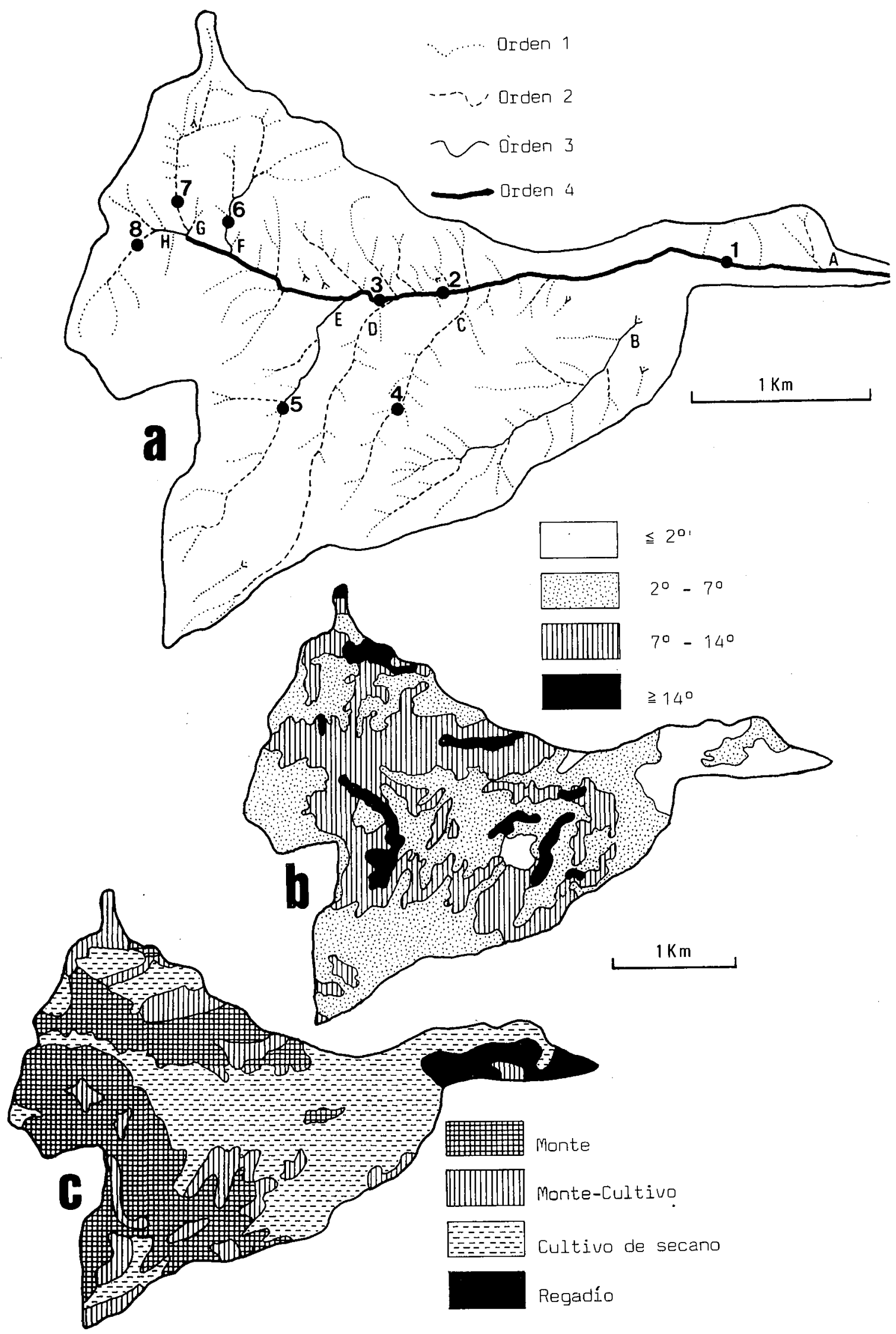

Figura 1. A) Red de drenaje, nomenclatura de las principales cuencas (letras) y ubicación de las estaciones de cálculo de caudal (números). B) Mapa de pendientes. C) Mapa de usos del suelo. 


\section{LLUVIAS TORRENCIALES Y CAMBIOS GEOMORFOLOGICOS}

Aparte de litologías, pendientes y morfología de la red de drenaje, otro elemento directamente relacionado con la escorrentía es el uso del suelo. En la cuenca existen cuatro tipos básicos (regadío, cultivos arbóreos y herbáceos de secano y monte). El regadío se ciñe exclusivamente a la zona baja (fig. 1C) y en superficie supone únicamente el $4,1 \%$. Los cultivos arbóreos de secano ocupan $1 / 3$ de la extensión total y se sitúan en el fondo de los valles y algunas laderas labradas en materiales terciarios, en la cuenca media. Los cultivos herbáceos de secano tienden a localizarse en las partes altas de la cuenca, sobre los glacis villafranquienses, en ocasiones como parcelas aisladas entre amplias extensiones de monte ${ }^{2}$. Por último, el monte ocupa cerca del $40 \%$ de la superficie y está constituido esencialmente por monte bajo, si exceptuamos pequeñas extensiones de repoblación forestal con Pinus halepensis; las especies predominantes en el monte bajo son Ulex parviflorus, Quercus coccifera, Rhamnus lycioides, Rosmarinus officinalis y Juniperus oxycedrus, todas ellas características del piso termomediterráneo. El grado de cobertura vegetal no es homogéneo: bastante elevado en las escasas zonas de repoblación, débil o moderado en las áreas de monte bajo (debido a la delgadez de los suelos y al carácter xérico de la región) y muy débil en las zonas agrícolas.

Los segmentos de cauce que conforman la red de drenaje de la cuenca (fig. 2) no están caracterizados por una morfología común. Por el contrario pueden llegar a diferenciarse hasta cuatro tipos básicos:

- El primero de ellos es el que constituye el último tramo del curso principal. El canal está encajado en depósitos aluviales recientes, parte de los cuales constituyen el cono de deyección final. Es de paredes subverticales y fondo plano; la relación profundidad/anchura decrece en sentido de la corriente, siendo algo mayor que la unidad en la parte superior.

- Los valles de fondo plano, sin cauce definido, caracterizan al resto del curso principal y parte baja de sus afluentes más importantes. Esta morfología es debida a la colmatación de un cauce previo por aportes aluviales y coluviales, sueltos y de textura fina predominante. Al mismo tiempo estos fondos de valle han sido utilizados en su totalidad como tierras de cultivo, mediante la concatenación de bancales cuyos muros (paredes de piedra asentada en seco) se disponen transversales al sentido del flujo, de un extremo a otro. Ello condiciona la inexistencia de un canal definido. Las pendientes longitudinales del valle son moderadas, $1,8^{\circ}$ en el principal y entre $2^{\circ}$ y $3^{\circ}$ en sus afluentes, lo que permite ir acumulando el desnivel en las paredes de los bancales para disponer de amplios espacios llanos.

- Un tercer tipo morfológico es el de los valles en $\mathrm{V}$ que se extienden justo aguas arriba del tipo anterior. Están labrados siempre sobre roquedo

2. A efectos cartográficos este tipo se ha asociado a los cultivos arbóreos o ha formado una categoría mixta con el monte, dada su intensa asociación. 
resistente (caliza), materiales que constituyen el fondo de los cauces. Los canales son estrechos y de fondo rocoso (calizas y calcoarenitas) y tienen un perfil longitudinal más abrupto, entre $3^{\circ}$ y $8^{\circ}$. Estas características son el resultado de su posición en la zona de tránsito entre el nivel villafranquiense de las divisorias y la zona central de vaciado cuaternario.

- Por último, sobre las rampas villafranquienses se sitúa un nuevo tipo de cauces que constituyen la cabecera de la mayoría de las cuencas. Son suaves concavidades que ondulan la topografía original de este nivel. La pendiente longitudinal es muy homogenea, entre $4,5^{\circ}$ y $6^{\circ}$.

\section{Lluvias y caudales}

Las lluvias caídas en la región de Ayora durante el temporal de octubre de 1982 alcanzaron volúmenes entre 360 y $581 \mathrm{~mm}$. en las estaciones situadas en un radio de $17 \mathrm{~km} .{ }^{3}$ (vid. PEREZ CUEVA y ARMENGOT, 1983). En la cuenca del B. de la Cuesta de la Vega es de suponer que las lluvias superaron los $450 \mathrm{~mm}$. Estos valores son muy elevados debido a la excepcionalidad del temporal y a que la región quedó situada en su centro álgido. Las intensidades de lluvia pueden extrapolarse a partir de la banda de pluviógrafo de la Central Nuclear de Cofrentes (fig. 3), salvando las dificultades de lectura derivadas de su periodicidad semanal. Se deduce que un $65 \%$ de la lluvia cayó entre las 7 y $12 \mathrm{~h}$. del día 20 , con intensidades máximas de 95 $\mathrm{mm} / 150 \mathrm{~min}$. (de $9 \mathrm{~h}$. a 11 h. 30') y $110 \mathrm{~mm} . / 60 \mathrm{~min}$. (de 7 h. a 8 h.). Con todo hay que destacar la persistencia de las fuertes intensidades (al menos durante 5 horas), hecho que diferencia en la región a los temporales de levante frente a las tormentas convectivas veraniegas.

La recurrencia de las lluvias torrenciales para esta área, según ELIAS CASTILLO (1977) se sitúa en torno a los $70 \mathrm{~mm} / \mathrm{día}$, con un período de retorno de 10 años, o $160 \mathrm{~mm} /$ día en cien años. Ello implica una recurrencia superior a los 6.000 años para el volumen de agua precipitada en Ayora durante este temporal. Estos valores son cuestionados por PEREZ CUEVA y ARMENGOT (1983) para quienes la recurrencia sería tan sólo secular.

Las lluvias produjeron tanto en la cuenca de estudio como en el conjunto de la cuenca del Júcar caudales elevados. La inexistencia de estaciones de aforo ha obligado a una estimación de los débiles máximos instantáneos a partir de la ecuación de Manning. Los cálculos se han realizado para 8 secciones de cauce situadas en 5 de los principales afluentes y en el colector (fig. 1A). En los primeros se han buscado lugares donde la sección de mojado no presentase modificaciones antrópicas, mientras que en el colector hemos eludido los puntos donde se produjo excavación o agradación importantes. La referencia utilizada para determinar la sección de mojado ha sido los restos de vegetación atrapados en las plantas que colonizaban el canal. El 


\section{LLUVIAS TORRENCIALES Y CAMBIOS GEOMORFOLOGICOS}

aspecto más problemático en el uso de la ecuación fue determinar los coeficientes de rozamiento de cada canal (vid. tablas en GARDINER y DACOMBE, 1983, pp. 142-143).

En todos los valles de la cuenca las aguas superaron el lecho en sentido estricto; los resultados obtenidos con la ecuación de Manning (tabla II) muestran la extraordinaria magnitud de los flujos. Debido a las características del método utilizado es necesaria una reflexión sobre los resultados. Estos, por sí mismos, son tan sólo una aproximación a la realidad, si bien concuerdan con las cantidades de precipitación medidas, al tiempo que son semejantes a los caudales obtenidos en otras áreas mediterráneas con condiciones de torrencialidad casi idénticas (vgr. Norte de Génova, GUIDO, 1973); el orden de magnitud entre los distintos volúmenes de agua calculados es probablemente válido.

TABLA II

Características relacionadas con la escorrentía aguas arriba de las secciones de estimación de caudal

\begin{tabular}{|c|c|c|c|c|c|c|c|c|c|c|}
\hline & Areal & Monte ${ }^{2}$ & Cultivo $^{2}$ & Dd & $\mathrm{Re}$ & $\mathrm{Rr}$ & $\mathrm{Rb}$ & $\begin{array}{l}\text { Pen- } \\
\text { diente }^{3}\end{array}$ & $\begin{array}{l}\text { Caud. } \\
\text { Abs. }{ }^{4}\end{array}$ & $\begin{array}{l}\text { Caud. } \\
\text { Esp. }\end{array}$ \\
\hline 1 & 6,00 & 39,0 & 46,7 & 6,2 & 0,76 & 71,5 & 5,0 & 7,92 & 121,8 & 20,0 \\
\hline 2 & 3,6 & 42,0 & 42,1 & 5,6 & 0,87 & 82,5 & 4,1 & 8,5 & 91,6 & 24,7 \\
\hline 3 & 2,7 & 44,0 & 41,5 & 5,7 & 0,82 & 84,9 & 3,8 & 8,9 & 57,8 & 21,16 \\
\hline 4 & 0,17 & 67,7 & 0,0 & 6,8 & 0,51 & 71,4 & 6,0 & 7,5 & 1,9 & 11,2 \\
\hline 5 & 0,41 & 72,5 & 3,9 & 4,5 & 0,57 & 100,3 & 4,0 & 7,98 & 15,9 & 38,2 \\
\hline 6 & 0,322 & 20,9 & 46,3 & 6,7 & 0,86 & 125,6 & 2,6 & 9,5 & 6,2 & 19,4 \\
\hline 7 & 0,29 & 27,9 & 31,6 & 7,4 & 0,62 & 131,3 & 10,0 & 9,2 & 7,6 & 25,6 \\
\hline 8 & 0,323 & 93,7 & 5,5 & 3,5 & 0,78 & 93,9 & 3,0 & 5,2 & 4,17 & 12,9 \\
\hline
\end{tabular}
1) $\mathrm{km}^{2}$
2) $\%$
3) grados sex.
4) $\mathrm{m}^{3} / \mathrm{seg}$.
5) $\mathrm{m}^{3} / \mathrm{seg} / \mathrm{km}^{2}$

Es lógico suponer que estos caudales estén determinados por diferentes factores y con este fin se han establecido en cada cuenca algunas correlaciones entre los caudales absoluto y específico calculados y algunas características de la cuenca aguas arriba del punto de medición. Estas características son la pendiente media de las laderas de la cuenca, el porcentaje de superficie dedicada a cultivos de secano y a monte y algunos índices morfométricos considerados significativos, como Dd, Re, Rb y Rr. Debido a las características de los datos el coeficiente de correlación considerado como más adecuado ha sido (tau) de Kendall (vid. tabla III). En contra de lo que teóricamente se suponía apenas se han encontrado relaciones significativas entre estos factores y el volumen máximo de agua evacuado en cada zona. 
Unicamente la superficie cultivada mantiene una correlación alta con los caudales absolutos $(0,57$, con $3,1 \%$ de aleatoriedad) pero claramente influida por el predominio de la dedicación agrícola en las áreas de fondo de cauce y especialmente en el principal, que a su vez reúne los mayores caudales (si se excluyen estas secciones de mojado la correlación baja a 0,2 ). Las relaciones de los caudales con los índices morfométricos no alcanzan niveles de confianza suficientes y únicamente la razón de relieve, con una relación positiva de 0,6 (para cuencas afluentes), nos señala la importancia que el desnivel general de la cuenca puede tener en los flujos. El que índices de jerarquización de la red de drenaje (Dd y Rb), índices de circularidad de las cuencas (re) y factores de escorrentía en ladera (pendiente y usos del suelo) no hayan mostrado apenas relación con el volumen de agua máximo alcanzado puede interpretarse en principio como resultado de la insuficiencia de datos para poder establecer correlaciones significativas. Ahora bien, la existencia de una buena correlación en los casos restantes permiten suponer que una población mayor no aumentaría el grado de correlación a la vez que aumentaría el grado de confianza del test. De ser cierta esta suposición pensamos que puede ser válida la hipótesis de que en estas condiciones de precipitación intensa y prolongada, cuando se alcanzan débitos máximos los factores de escorrentía en las laderas y la morfología de la red no ejercen control sobre los caudales. La confirmación de esta hipótesis necesita, no obstante, de estudios más detallados que han de partir de hidrogramas elaborados a partir de estaciones de aforos.

\section{TABLA III}

Valores de correlación (test ) entre caudales absolutos y específicos y características de las cuencas.

a) Para las ocho secciones de estimación de caudal:

$\begin{array}{lcccccccc} & \text { Area } & \text { Monte } & \text { Cultivo } & \mathrm{Dd} & \mathrm{Re} & \mathrm{Rr} & \mathrm{Rb} & \text { Pendien. } \\ \text { Caudal Absoluto } & 0,79 & -0,21 & 0,57 & -0,07 & 0,29 & -0,21 & 0,14 & 0,0 \\ \text { Caudal específico } & 0,21 & -0,07 & 0,0 & -0,21 & 0,0 & 0,36 & 0,14 & 0,29\end{array}$

b) Para las cinco secciones de estimación de caudal correspondientes a los afluentes:

$\begin{array}{ccccccccc} & \text { Area } & \text { Monte } & \text { Cultivo } & \mathrm{Dd} & \mathrm{Re} & \mathrm{Rr} & \mathrm{Rb} & \text { Pendien. } \\ \text { Caudal específico } & 0,4 & 0,0 & 0,2 & 0,0 & 0,0 & 0,6 & 0,0 & 0,2\end{array}$

3. Ayora (escuelas) $41 \mathrm{~mm}$. Ayora (SMN) $382 \mathrm{~mm}$. Ayora (La Hunde) $\geq 431 \mathrm{~mm}$., Teresa de Cofrentes $360 \mathrm{~mm}$., Jalance 546, Cofrentes (Central Nuclear) $581 \mathrm{~mm}$., Pico Caroig $\geq$ $500 \mathrm{~mm}$. 


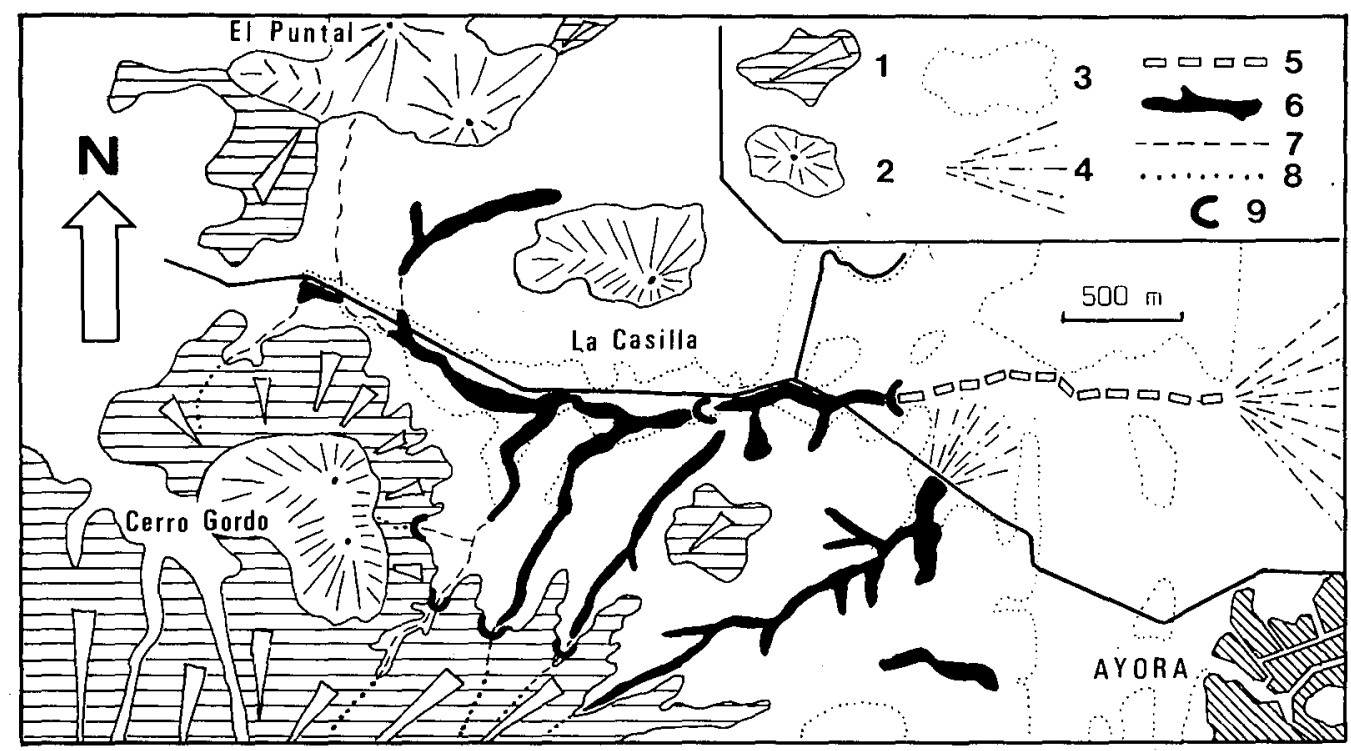

Figura 2. Esquema geomorfológico:1) Superficie villafranquiense 2) Relieves sobre, la superficie villafranquiense 3) Depósitos cuaternarios 4) Zonas de divergencia de flujo 5) Cauces encajados sobre depósitos cuaternarios 6) Valles de fondo plano 7) Valles en "V" 8) Cauces sobre la superficie villafranquiense 9) Cascada.

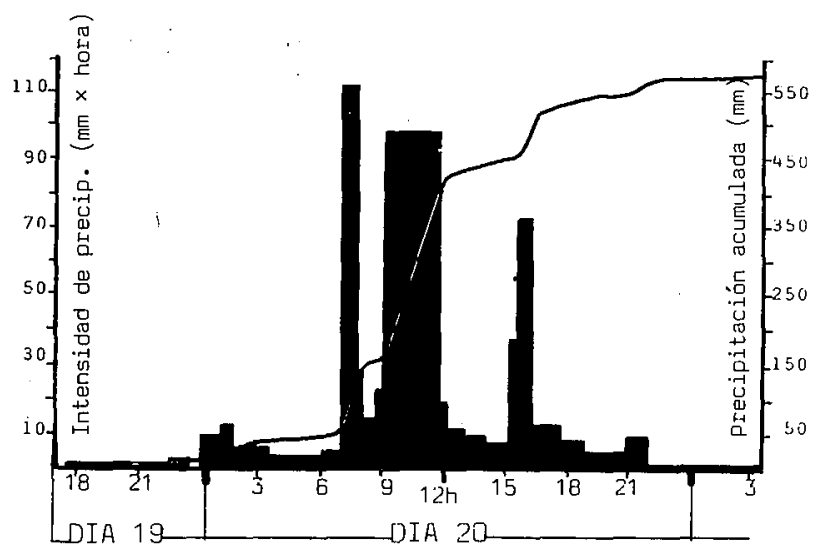

Figura 3. Histograma de intensidades de lluvia y curva acumulada de precipitación en la tormenta de los días 1921 de octubre de 1984. Central Nuclear de Cofrentes.

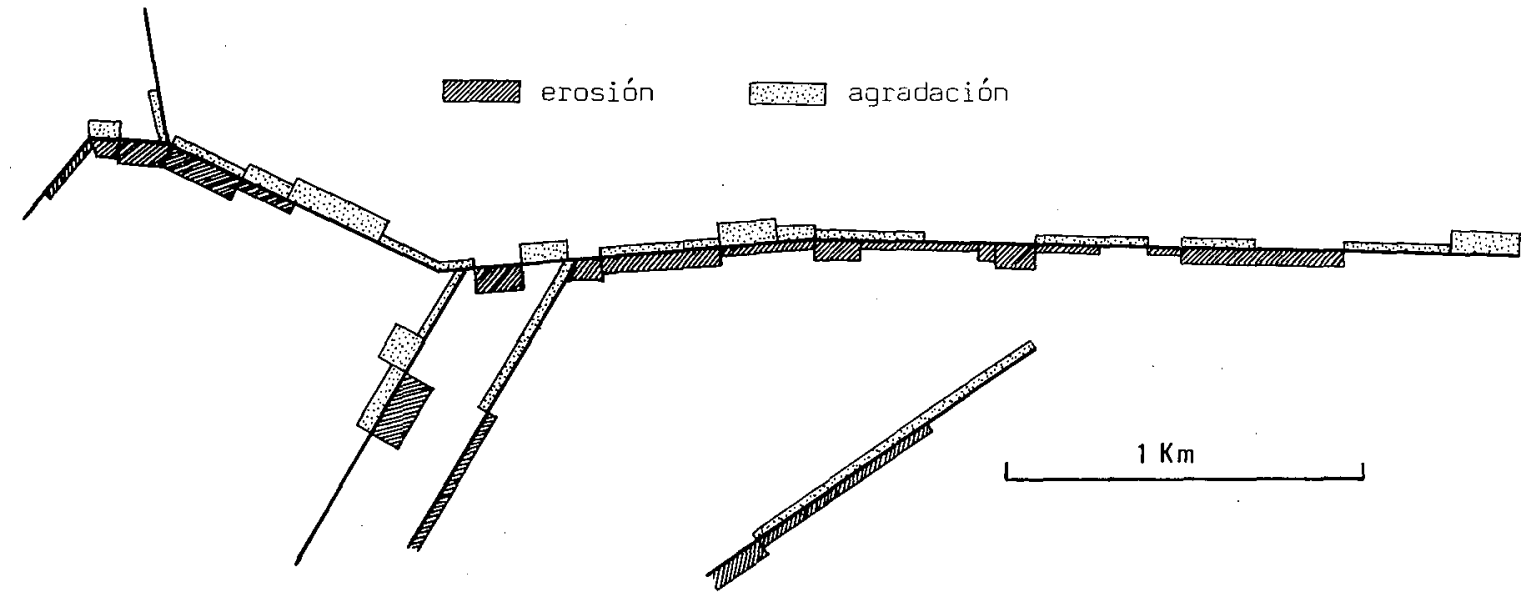

Figura 4. Esquema de zonas de erosión y agradación producidas en el B. de la Cuesta de la Vega durante la tormenta de los días 19-21 de octubre de 1982 . 


\section{ALEJANDRO PEREZ CUEVA, ADOLFO CALVO CASES}

\section{Formas y procesos en los canales}

Los flujos extraordinarios resultantes del temporal de octubre de 1982 permitieron una reactivación momentanea, pero eficaz, de los procesos geomórficos y de modo especial de los procesos fluviales de erosión y deposición. Esta activación se extendió a todos los valles de la cuenca media y baja del río Júcar, pero los cambios geomorfológicos de mayor relevancia ocurrieron en los cauces de las cabeceras que, como el Bco. de la Cuesta de la Vega, son formas no adaptadas aún a las características del sistema fluvial en el que se integran (CALVO y FUMANAL, 1983, pp. 116-119).

La figura 4 es un esquema que pondera la magnitud de los procesos de erosión y deposición en distintos segmentos de cauce a lo largo de la cuenca. Los sectores de cauce más afectados por la erosión corresponden a la parte alta y media del principal y a la parte media de algunos afluentes, que coincide con la entrada de las aguas en la zona de valles de fondo plano. Por su parte, la agradación alcanza cotas más elevadas en los tramos inmediatos a la erosión así como en el último tramo del cauce principal, donde se produce una difluencia de las aguas. No obstante, aunque con valores bajos, hay que destacar su presencia a lo largo de todos los segmentos de cauce.

Los fenómenos de erosión más relevantes han sido la nueva formación de cauces, la rotura de paredes de los bancales, el inicio de acarcavamientos a partir de estos puntos de rotura, la formación de grandes surcos de arroyada, la remoción de la capa de labranza en los bancales de fondo de valle y la zapa lateral de las paredes en los tramos de cauce preexistentes. Durante la crecida, las paredes de los bancales se convirtieron en cascadas, bajo las cuales el movimiento turbillonar del agua formó pilancones. Si la profundidad del agua es uniforme en todo el fondo del valle este pilancón no es mas que un pequeño canal paralelo al pie de toda la pared, pero cuando existen factores que fomentan, bien el incremento de la profundidad del agua, bien el incremento de su velocidad a lo largo de una línea, se incrementa también en ese punto la acción de la zapa basal hasta que se abre una brecha en la pared. Estas roturas de paredes se han producido en casi todos los casos y en muchos de ellos ha progresado una acarcavamiento hacia el interior del bancal por retroceso del pilancón. La formación de un nuevo cauce encajado sobre la superficie anterior es el resultado de la concatenación de diversas roturas y cárcavas. Estos cauces nuevos tienen una forma en sección transversal muy característica: son pequeños canales de márgenes verticales o incluso en contrapendiente, de anchura muy variable y profundidad entre 1 y $2 \mathrm{~m}$., que recuerdan las formas de los arroyos americanos descritos por LEOPOLD (1978) y COOKE y REEVES (1976). El perfil longitudinal es también muy irregular, con proliferación de pilancones y escalones; estos últimos relacionados con distintos tipos de cohesión en el fondo. 


\section{LLUVIAS TORRENCIALES Y CAMBIOS GEOMORFOLOGICOS}

En los sectores de cauce preexistente, el proceso de erosión dominante ha sido la zapa lateral sobre las márgenes. Esta fue activa tanto en las concavidades de meandro como en los tramos rectos, lo que ha traído como consecuencia el ensanche del cauce, de modo especial en la base cuando las márgenes originales tenían pendientes más suaves. varios:

Los procesos sedimentarios operados durante la avenida han sido

a) En primer lugar se han producido algunas acumulaciones al principio de la crecida y previas a la excavación de un nuevo cauce, ya que aparecen seccionadas por éste. Se trata de capas de cantos y gravas subangulosos abandonados sobre la superficie original del fondo de valle, en lugares de ruptura de pendiente, especialmente en las cabeceras.

b) Durante la avenida se han producido acumulaciones muy poco seleccionadas producto de procesos rápidos de carga y descarga. Estas acumulaciones caóticas se ubican inmediatamente aguas abajo de los tramos de máxima degradación. En los lugares donde no se ha producido más que un inicio de acarvamiento existen acumulaciones similares pero menos potentes situadas a la salida de los pilancones de pie de cascada.

c) A lo largo de casi todo el cauce existen depósitos de final de avenida, caracterizados por una mayor homometría y menor tamaño de las partículas, con predominio de gravas y arenas. Estas acumulaciones se disponen libremente, en forma de barras de canal longitudinales (longitudinal bars, MIALL, 1977), o se elaboran a partir de obstáculos como grandes bloques o árboles (acumulaciones tipo current shadows, ALLEN, 1982). En la cuenca baja la dinámica sedimentaria ha dado lugar a la formación de barras de meandro (point bars) de características texturales semejantes a las anteriores.

d) Por último, en la zona de difluencia de aguas del final de la cuenca se ha producido una importante acumulación de materiales. Por su heterometría puede deducirse un aporte continuo de sedimentos a lo largo de toda la crecida, si bien no se pueden distinguir estructuras sedimentarias debido a la removilización antrópica posterior.

\section{Factores de los procesos de erosión}

1) Factores relativos a la magnitud de los flujos: El único claro, aparte de las variaciones locales de la torrencialidad, es el tamaño de las cuencas de drenaje. Otros, como $\mathrm{Db}, \mathrm{Rb}$ y $\mathrm{Re}$, parece que no han tenido influencia en la escorrentía, así como los distintos usos del suelo o las características litológicas de cada cuenca. 
2) Factores que determinan el tipo de flujo: Los tramos con incisión tienen por lo general una mayor pendiente media que los inmediatos, en los que ha dominado la agradación. El factor pendiente local, a través de la velocidad y turbulencia del flujo y por tanto de su agresividad ha influido, pues, notablemente.

- Asimismo, existe una relación inversa entre la anchura de los valles de fondo plano y los síntomas de erosión, factores que han anulado incluso la influencia de la pendiente. En los tramos más anchos (más de $20 \mathrm{~m}$.) la disminución del radio hidráulico y la consecuente disminución de velocidad parecen haber determinado la ausencia de incisión, si bien en los campos de cultivo no abandonados la capa de labranza ha sido removida en su totalidad. Por su parte los tramos con mayor incisión coinciden con valles en torno a los $10 \mathrm{~m}$. de ancho.

- También puede haber influido localmente, en las cabeceras de los valles de fondo plano, el que las aguas tuvieran gran capacidad de carga debido a que la cuenca de recepción inmediata estaba cubierta de vegetación natural, factor protector contra la erosión.

3) Factores relativos a la protección y resistencia del suelo contra la erosión. Han actuado unidos al estar vinculados al uso o abandono de los campos de cultivo. Los tramos de cauce con cultivos abandonados apenas han sufrido degradación, dada la protección que supone la vegetación espontánea y dado el mayor grado de cohesión del suelo.

4) Factores que propician los mecanismos de incisión: Las paredes de las terrazas son los lugares donde se han iniciado los procesos de incisión. La pared es el punto en el que se concentra la pendiente del valle tras la modificación antrópica y por tanto donde las aguas alcanzan la máxima energía cinética. Ello produce en primer lugar su zapa basal y tras un lapso de tiempo determinado por la energía de las aguas y la resistencia de la pared, su rotura. La cohesión de la pared, su pendiente y su altura son pues elementos importantes en el equilibrio energía cinética-resistencia. El primero retarda la rotura y por tanto el inicio de la incisión. La pendiente y la altura, por su parte pueden incrementar (paredes verticales y altas) o disminuir (inclinadas o bajas) la energía cinética de las aguas. En este caso los dos primeros factores actuaron positivamente (paredes consistentes e inclinadas $-45^{\circ}-$ ) mientras que el tercer factor lo ha hecho negativamente ya que el tipo de abancalamientos propiciaba desniveles de hasta 2,50 m.

\section{El factor antrópico}

Queda por cuestionarse finalmente la trascendencia de la intervención humana en este medio y la pregunta clave es si el hombre, alterando la geo- 


\section{LLUVIAS TORRENCIALES Y CAMBIOS GEOMORFOLOGICOS}

metría de los fondos de valle ha incrementado el impacto erosivo de las lluvias del temporal.

Los aterrazamientos son considerados en general como prácticas de protección mecánica de los suelos bastante efectiva (NIR, 1983), en especial las channel bottom terraces. Cumplen dos de los objetivos definidos por BRYAN (1979) para las medidas de conservación del suelo: incrementan la capacidad de infiltración de las tierras y reducen la velocidad del flujo superficial. Sin embargo como en cualquier otra técnica es difícil ajustar su diseño para que no produzca desequilibrios mayores. Comúnmente se sugiere su uso combinándolo con acequias estabales de derivación de las aguas de escorrentía (HUDSON, 1982; BRYAN, 1979) y se ha llegado a calcular su altura óptima mediante ecuaciones. En la cuenca estudiada no se ha aplicado un sistema de protección combinado de terrazas acanaladas o de simples canales laterales de derivación de aguas, como se efectúa en otros lugares de la región valenciana. La forma de los muros, por su parte obedece, sin duda, a la tradición cultural; en este sentido es de notar que la forma inclinada, especialmente resistente, es una característica casi exclusiva de la región de Ayora y S de la Requena (PEREZ CUEVA, 1982). Con todo hemos de retomar la idea de la extraordinaria magnitud de los flujos, que llegaron a colmatar todos los fondos de valle. En tales condiciones es difícil pensar en la efectividad, incluso, de un sistema de protección completo y científico y concluir que la acción humana condicionaría más el tipo de mecanismo de los procesos erosivos que su propia magnitud. 


\section{BIBLIOGRAFIA}

ALLEN, J.R.L. (1982), Sedimentary structures, 2 vols., Elsevier Scient. Publ. Comp., Amsterdam.

BRYAN, R.B. (1979). "Soil erosion and conservation", en Man and environmental processes, Gregory, K.J. y Walling, D.E., Dawson Westview Press, Folkestone, pp. 207-221.

CALVO CASES, A. y FUMANAL GARCIA, M.P. (1983), "Repercusiones geomorfológicas de las lluvias torrenciales de octubre de 1982 en la cuenca media del río Júcar", Cuad. de geogr., 32-33, Valencia, pp. 101-120.

COOKE, R.U. y REEVES, R.W. (1976), Arroyos and Environmental Change in American South-West, Clarendon press, Oxford, 213 p.

CHRISTOFOLETTI, A. (1971), "Analisis morfometrica das bacias hidrograficas", Bol. Geogr., pp. 131-159.

Elias CASTILlo, F. (1979). Precipitaciones máximas en España, Ministerio de Agricultura-ICONA, Madrid, $545 \mathrm{p}$.

GARDINER, V. y DACKOMBE, R. (1983), Geomorfological Field Manual, George Allen \& Unwin, London, 254 p.

GUIGO, M. (1973). "Pluie et crue des 7 et 8 octuibre 1970 dans la region genoise", Méditerranée, pp. 55-80.

HUDSON, N. (1982). Conservación del suelo, Edit. Reverté. Barcelona, 335 p.

LEOPOLD, L.B. (1978). "El Asunto del Arroyo" en EMBLETON, C. et al., Geomorphology Present Problems and Future Prospects, Oxford University Press, pp. 25-39.

MIALL, A.D. (1977). "A review of the braided river depositional environment", Earth Sci. Rev., 13, pp. 1-62.

NIR, D. (1983). "Man, ageomorphological agent", Keter Publ. House. Jerusalem, $165 \mathrm{p}$.

PEREZ CUEVA, A. (1982). "Erosión acelerada antrópica”, Inst. Alfons el Magnànim, Dip. Prov. Valencia, inédito, $110 \mathrm{ff}$.

PEREZ CUEVA, A.J. y ARMENGOT SERRANO, R. (1983). "El temporal de octubre de 1982 en el marco de las lluvias torrenciales en la cuenca baja del Júcar", Cuad. de Geogr., 32-33, pp. 61-86, Univ. Valencia. 